Publ. RIMS, Kyoto Univ.

19 (1983), 493-518

\title{
Preparatory Structure Theorem for Ideals Defining Space Curves
}

By

\author{
Mutsumi AMASAKI*
}

\section{Introduction}

In the study of $\mathrm{Hilb}\left(\mathbb{P}_{k}^{3}\right)$ any knowledge of concrete structure of ideals defining the minimal cone of a curve in $\mathbb{P}_{k}^{3}$ would benefit one greatly. Here 'curve' means an equidimensional complete scheme over a field $k$ of dimension one. Let $I \subset R:=k\left[x_{1}, x_{2}, x_{3}, x_{4}\right]$ be the ideal defining the ninimal cone of a curve $X \subset \mathbb{P}_{k}^{3}$. Then we know that $\operatorname{dim} R / I$ $=2$ and $\operatorname{depth}_{\mathfrak{m}} R / I \geqq 1$. The present paper is aimed at giving a way to describe all homogeneous ideals with this property. We show in Section 3 that any such ideal, with a free resolution for it, is determined by a matrix of special type which satisfies seemingly a simple relation (See Proposition 3.1, Corollary 3.5 and Theorem 3.7). We discuss briefly the easiest case in Section 4 to illustrate how the results of Section 3 work.

In order to provide necessary techniques for obtaining our main results, we describe in Section 2 a general method to compute a free resolution for any ideal in $k\left[\left[x_{1}, \cdots, x_{n}\right]\right]$. The free resolutions indicated there start from the generalised Weierstrass preparation theorem due to $\mathrm{H}$. Grauert and H. Hironaka. The author borrowed this setting from [8].

\section{Notation}

1. $k$ denotes an infinite field with arbitrary characteristic from Section 1 to Section 3 .

2. Let $A$ be $k\left[\left[t_{1}, \cdots, t_{d}\right]\right]$ or $k\left[t_{1}, \cdots, t_{d}\right], \mathfrak{n}$ its maximal ideal gener-

Communicated by S. Nakano, February 9, 1982.

* Research Institute for Mathematical Sciences, Kyoto University, Kyoto 606, Japan. 
ated by $t_{1}, \cdots, t_{d}$, and $x_{1}, \cdots, x_{n}$ indeterminates over $\Lambda$. We set

$$
\begin{aligned}
& A((i, n))=A\left[\left[x_{i+1}, \cdots, x_{n}\right]\right] \\
& A(i, n)=A\left[x_{i+1}, \cdots, x_{n}\right]
\end{aligned}
$$

both for $0 \leqq i \leqq n$. In particular $A((n, n))=A(n, n)=A$.

3. $A((i, n))^{p}$ or $A(i, n)^{p}$ denotes the set of column vectors unless otherwise specified.

4. Let $B_{1}, B_{2}, \cdots, B_{s}$ be arbitrary $A$-modules. We denote by $B_{1} \oplus B_{2} \oplus \cdots$ $\oplus B_{s}$ or by $\bigoplus_{i=1}^{s} B_{i}$ the $A$-module

$$
\left\{\left(\begin{array}{c}
b_{1} \\
b_{2} \\
\vdots \\
b_{s}
\end{array}\right) \mid b_{i} \in B_{i} \quad \text { for } \quad 1 \leqq i \leqq s\right\}
$$

5: For an $f \in A((i, n))^{p}$ (resp. $\left.f \in A(i, n)^{p}\right) \quad f(0)$ denotes $f(\bmod \mathfrak{n})$ which is naturally thought of as an element of $k((i, n))^{p}$ (resp. $\left.k(i, n)^{p}\right)$ via $k G A$.

6. $1_{p}$ denotes $p \times p$ identity matrix.

7. For an $f=\sum_{\mid \nu_{i}^{\prime}=0}^{\infty} a_{\nu} x^{\nu} \in A((i, n))$ with $a_{\nu} \in A, o(f)=\min \left\{|\nu| \mid a_{\nu} \neq 0\right\}$, and in $(f)=\sum_{|\nu|=0(f)} a_{\nu} x^{\nu}$.

8. $\boldsymbol{Z}_{0}=\{\alpha \in \boldsymbol{Z} \mid \alpha \geqq 0\}$.

\section{$\S 1$. Preliminaries}

Let $A$ be a formal power series ring over a field $k, \mathfrak{n}$ its maximal ideal, and $x_{1}, \cdots, x_{n}$ indeterminates over $A$.

Definition 1.1. Let $\bar{a}=\left(a_{1}, \cdots, a_{p}\right)$ be a sequence of integers. For each $f=\left(f_{i}\right) \in A((0, n))^{p}$ we define

$$
d_{\bar{a}}(f)=\min _{1 \leqq i \leqq p}\left(o\left(f_{i}\right)+a_{i}\right) .
$$

Suppose we are given a set of positive integers

$$
\left\{m, l_{1}, \cdots, l_{m}, l=\sum_{i=1}^{m} l_{i}, s_{\alpha}=\sum_{i=\alpha+1}^{m} l_{i}(0 \leqq \alpha \leqq m-1)\right\},
$$

a sequence of integers $\bar{q}=\left(q_{1}, \cdots, q_{l}\right)$, and $l \times s_{\alpha}$ matrices $\chi^{\alpha}(1 \leqq \alpha \leqq m-1)$ 
with entrics in $\Lambda(((), n))$ satisfying the following two conditions:

(I) Write $\chi^{\alpha}=\left(\chi_{1}^{\alpha}, \cdots, \chi_{s_{o}}^{\alpha}\right)(1 \leqq \alpha \leqq m-1)$, then each column vector $\chi_{\beta}^{\alpha}$ is in $\bigoplus_{i=1}^{m} A((i-1, n))^{l_{i}}$.

(II) $\quad d_{\bar{q}}\left(\chi_{j}^{\alpha}\right) \geqq 1+q_{l-s_{\alpha}+j}$ for $1 \leqq \alpha \leqq m-1,1 \leqq j \leqq s_{\alpha}$.

We set

$$
\begin{aligned}
\psi^{\alpha}=\left(\psi_{1}^{\alpha}, \cdots, \psi_{s_{\alpha}}^{\alpha}\right)= & \left(\begin{array}{c}
0 \\
x_{\alpha} 1_{s_{\alpha}}
\end{array}\right) \begin{array}{l}
l \frac{\hat{i}}{\downarrow} s_{\alpha} \\
\downarrow
\end{array} \\
& \cdots s_{\alpha} \cdots \rightarrow
\end{aligned}
$$

for $1 \leqq \alpha \leqq m-1$.

\section{Proposition 1.2.}

1) $\quad \Lambda((0, n))^{l}=\left\{\bigoplus_{\alpha=1}^{m-1} \bigoplus_{j=1}^{s_{\alpha}} \psi_{j}^{\alpha} A((\alpha-1, n))\right\} \oplus\left\{\stackrel{\oplus}{\oplus}_{i=1}^{m} A((i-1, n))^{l_{i}}\right\}$

(direct sum as A-modules).

2) If $f=\sum_{\alpha=1}^{m-1} \sum_{j=1}^{s_{\alpha}} \psi^{\alpha} g_{j}^{\alpha}+h$ reith $g_{j}^{\alpha} \in A((\alpha-1, n))$ and $h \in \bigoplus_{i=1}^{m} A((i-1, n))^{l_{i}}$. then

$$
\left\{\begin{array}{l}
d_{\bar{q}}(f) \leqq d_{\bar{q}}\left(\psi^{\alpha}\right)+o\left(g_{j}^{\alpha}\right) \quad \text { for } \quad 1 \leqq \alpha \leqq m-1,1 \leqq j \leqq s_{\alpha} \\
d_{\bar{q}}(f) \leqq d_{\bar{q}}(h)
\end{array}\right.
$$

Proof. 1) is a consequence of the following:

$(1)_{\mu}$

$$
\begin{aligned}
A((0, n))^{l}= & \left\{\bigoplus_{\alpha=1}^{\mu} \psi^{\alpha} A((\alpha-1, n))^{s_{\alpha}}\right\} \\
& \oplus\left\{\stackrel{\leftrightarrow}{\oplus=1}_{i=1}^{\mu+1} A((i-1, n))^{l_{i}} \oplus A((\mu, n))^{s_{\mu+1}}\right\}
\end{aligned}
$$

for $1 \leqq \mu \leqq m-1$, where $s_{m}=0$.

Let $\psi^{\alpha 1}\left(\right.$ resp. $\left.\chi^{\alpha 1}\right)$ be the $s_{\alpha} \times s_{\alpha}$ matrix consisting of lower $s_{\alpha}$ rows of $\psi^{\alpha}$ (resp. $\left.\chi^{\alpha}\right)$ and $\psi^{\alpha 0}$ the upper $l-s_{\alpha}$ rows of $\psi^{\alpha 1}$ for $1 \leqq \alpha \leqq m-1$. Then, to prove (1) ", we need a lemma.

\section{Lemma 1.3.}

$$
\Lambda((\alpha-1, n))^{s_{a}}=\psi^{\alpha 1} A((\alpha-1, n))^{s_{\alpha}} \oplus A((\alpha, n))^{s_{\alpha}}
$$


for $1 \leqq \alpha \leqq m-1 . \quad$ (direct sum as A-modules)

Proof of Lemma 1.3. If $\psi^{\alpha 1} g+h=0$ with $g \in A((\alpha-1, n))^{s_{\alpha}}$, $h \in A((\alpha, n))^{s_{\alpha}}$, then

$$
\left(\operatorname{det} \psi^{\alpha 1}\right) g=-\left(\operatorname{det} \psi^{\alpha 1}\right)\left(\psi^{\alpha 1}\right)^{-1} h \text {. }
$$

We observe that $\operatorname{det} \psi^{\alpha 1}=x_{\alpha}^{s_{\alpha}}+\sum_{i=1}^{s_{\alpha}-1} \phi_{i} x_{\alpha}^{i}$, where $\phi_{i} \in A((\alpha, n))$ and $o\left(\phi_{i} x_{\alpha}^{i}\right)$ $\geqq s_{\alpha}$ by (II), and that the degree of each component of $-\left(\operatorname{det} \psi^{\alpha 1}\right)\left(\psi^{\alpha 1}\right)^{-1} h$ with respect to $x_{\alpha}$ is strictly smaller than $s_{\alpha}$. From this and Weierstrass division theorem we deduce $g=h=0$. Thus the sum is direct. Next we show that each $f \in A((\alpha-1, n))^{s_{\alpha}}$ can be written $f=\psi^{\alpha 1} g+h$ with $g \in A((\alpha-1, n))^{s_{\alpha}}$ and $h \in A((\alpha, n))^{s_{\alpha}}$ for $1 \leqq \alpha \leqq m-1$. Write $f=$ $\sum_{t=0}^{\infty} x_{\alpha}^{t} a_{t}$ where $a_{t} \in A((\alpha, n))^{s_{\alpha}}$. We claim

$$
\left\{\begin{array}{l}
g=\sum_{t=1}^{\infty} \sum_{i=1}^{t} x_{\alpha}^{t-i}\left(\chi^{\alpha 1}\right)^{i-1} a_{t} \\
h=\sum_{t=0}^{\infty}\left(\chi^{\alpha 1}\right)^{t} a_{t}
\end{array}\right.
$$

are both well defined.

Proof of (1.4) With each matrix $X$ with entries in $A((0, n))$ we associate a matrix $\Delta(X)$ whose $(i, j)$ component is the order $o\left(x_{i j}\right)$ of the $(i, j)$ component $x_{i j}$ of $X$. Then we know by (II) that

$(i, j)$ component of $\Delta\left(\chi^{\alpha 1}\right) \geqq 1+q_{l-s_{\alpha}+j}-q_{l-s_{\alpha}+i}$

$$
\text { for } \quad 1 \leqq \alpha \leqq m-1, \quad 1 \leqq i, j \leqq s_{\alpha} .
$$

From this we get

$(i, j)$ component of $\Delta\left(\left(\chi^{\alpha 1}\right)^{p}\right) \geqq p+q_{l-s_{\alpha}+j}-q_{l-s_{\alpha}+i}$

$$
\text { for } 1 \leqq \alpha \leqq m-1, \quad 1 \leqq i, j \leqq s .
$$

Hence

$$
d_{\bar{q}(\alpha)}\left(\left(\chi^{\alpha 1}\right)^{p} a_{t}\right) \geqq p+d_{\bar{q}(\alpha)}\left(a_{t}\right) \quad \text { for } \quad p \geqq 0, t \geqq 0
$$

where $\bar{q}(\alpha)=\left(q_{l-s_{\alpha}+1}, \cdots, q_{l}\right)$. Therefore the formal sums in (1.4) are well defined.

$g \in A((\alpha-1, n))^{s_{\alpha}}, h \in A((\alpha, n))^{s_{\alpha}}$ by the definition and we see by 
an easy computation that $f^{\circ}=\psi^{\circ 1} g+h$. Thus Lemma 1.3 is proved.

Proof of (1) $\mu$ If $\mu=1$ we know from Lemma 1.3 that

$$
A((0, n))^{s_{1}}=\psi^{11} A((0, n))^{s_{1}} \oplus A((1, n))^{s_{1}} .
$$

From this

$$
\begin{aligned}
A((0, n))^{l}= & \psi^{1} A((0, n))^{s_{1}} \\
& \oplus\left\{\Lambda((0, n))^{l_{1}} \oplus A((1, n))^{l_{2}} \oplus A((1, n))^{s_{2}}\right\}
\end{aligned}
$$

is almost clear. So by induction we assume

$$
\begin{aligned}
A((0, n))^{l}= & \left\{\bigoplus_{\alpha=1}^{\mu_{0}} \psi^{\alpha} A((\alpha-1, n))^{s_{\alpha}}\right\} \\
& \oplus\left\{\bigoplus_{i=1}^{\mu_{0}+1} A((i-1, n))^{l_{i}} \oplus A\left(\left(\mu_{0}, n\right)\right)^{s_{\mu_{0}+1}}\right\}
\end{aligned}
$$

for some $\mu_{0} \geqq 1$. We have

$$
A\left(\left(\mu_{0}, n\right)\right)^{s_{\mu_{0}+1}}=\psi^{\mu_{0}+1} A\left(\left(\mu_{0}, n\right)\right)^{s_{\mu_{0}+1}} \oplus A\left(\left(\mu_{0}+1, n\right)\right)^{s_{\mu_{0}+1}}
$$

by Lemma 1.3. Since each column vector of $\psi^{\mu_{0}+10}$ is in $\overbrace{i=1}^{\mu_{0}+1} A((i-1, n))^{l_{i}}$, (3) implies that

$$
\begin{aligned}
& \bigoplus_{i=1}^{\mu_{0}+1} A((i-1, n))^{l_{i}} \oplus A\left(\left(\mu_{0}, n\right)\right)^{s_{\mu_{0}+1}} \\
& \quad=\psi^{\mu_{0}+1} A\left(\left(\mu_{0}, n\right)\right)^{s_{\mu_{0}-1}} \oplus\left\{{\stackrel{\oplus}{\mu_{0}+2}}^{\mu_{1}+2} A((i-1, n))^{l_{i}} \oplus A\left(\left(\mu_{0}+1, n\right)\right)^{s_{\mu_{0}+2}}\right\} .
\end{aligned}
$$

From (1) $\mu_{0}$ and (4) we get (1) $\mu_{\mu_{0}+1}$. Thus $(1)_{\mu}$ is obtained for $1 \leqq \mu$ $\leqq m-1$ and Proposition 1.2.1) is proved. Proposition 1.2.2) follows from (1.4), (2), and the proof of (1) $\mu$ Q.E.D.

Let $M$ be an $A((0, n))$-module which is a direct sum of $A$-modules $(*)$

$$
M=\bigoplus_{i=1}^{m} \bigoplus_{j=1}^{l_{i}} f_{l-s_{i-1}+j} A((i-1, n))
$$

where $f_{j} \in M$ for $1 \leqq j \leqq l$. We want to know the relation module

$$
M_{1}=\left\{\psi={ }^{t}\left(\psi_{1}, \cdots, \psi_{l}\right) \in A((0, n))^{l} \mid \sum_{i=1}^{l} \psi_{i} f_{i}=0\right\} .
$$

Since $M$ is an $A((0, n))$-module, $x_{\alpha} f_{j} \in M$ for any $1 \leqq \alpha \leqq n, 1 \leqq j \leqq l$, 
whence we may write for $1 \leqq \alpha \leqq m-1,1 \leqq j \leqq s_{\alpha}$

$$
x_{\alpha} f_{l-s_{\alpha}+j}=\sum_{i=1}^{m} \sum_{\beta=1}^{l_{i}} f_{l-s_{i-1}+\beta} \tilde{\chi}_{l-s_{i-1}+\beta, j}^{\alpha}
$$

with $\tilde{\chi}_{l-s_{i-1}+\beta, j}^{\alpha} \in A((i-1, n))$. We set

$$
\left\{\begin{array}{l}
\tilde{\chi}_{j}^{\alpha}={ }^{t}\left(\tilde{\chi}_{1 j}^{\alpha}, \cdots, \tilde{\chi}_{l j}^{\alpha}\right) \\
\tilde{\chi}^{\alpha}=\left(\tilde{\chi}_{1}^{\alpha}, \cdots, \tilde{\chi}_{s_{\alpha}}^{\alpha}\right) \quad \text { for } \quad 1 \leqq \alpha \leqq m-1 .
\end{array}\right.
$$

Then $\tilde{\chi}^{\alpha}(1 \leqq \alpha \leqq m-1)$ satisfy the condition (I) by their construction but (II) may not be guaranteed. In the situations we shall later encounter the condition (II) is also satisfied by $\tilde{\chi}^{\alpha}(0)$. Therefore we assume that $\tilde{\chi}^{\alpha}(0)$ satisfies the condition (II).

Put

$\left(\begin{array}{c}* * \\ * *\end{array}\right)$

$$
\left\{\begin{array}{c}
\widetilde{\psi}^{\alpha}=\left(\widetilde{\psi}_{1}^{\alpha}, \cdots, \widetilde{\psi}_{s_{\alpha}}^{\alpha}\right)=\left(\begin{array}{c}
0 \\
x_{\alpha} 1_{s_{\alpha}}
\end{array}\right)-\tilde{\chi}^{\alpha} \\
\psi^{\alpha}=\left(\psi_{1}^{\alpha}, \cdots, \psi_{s_{\alpha}}^{\alpha}\right)=\left(\begin{array}{c}
0 \\
x_{\alpha} 1_{s_{\alpha}}
\end{array}\right)-\tilde{\chi}^{\alpha}(0),
\end{array}\right.
$$

then $\widetilde{\psi}_{j}^{\alpha}-\psi_{j}^{\alpha} \in \mathfrak{n} A((0, n))^{l}$ for $1 \leqq \alpha \leqq m-1,1 \leqq j \leqq s_{\alpha}$.

First we have

Corollary 1.5.

1) $\quad A((0, n))^{l}=\left\{\bigoplus_{\alpha=1}^{m-1} \bigoplus_{j=1}^{s_{\alpha}} \widetilde{\psi}_{j}^{\alpha} A((\alpha-1, n))\right\} \oplus\left\{\bigoplus_{i=1}^{m} A((i-1, n))^{l_{i}}\right\}$

(direct sum as A-modules)

2) If $f=\sum_{\alpha=1}^{m-1} \sum_{j=1}^{s_{\alpha}} \widetilde{\psi}_{j}^{\alpha} g_{j}^{\alpha}+h$ with $g_{j}^{\alpha} \in A((\alpha-1, n))$ and $h \in$ $\stackrel{\oplus}{i=1}_{m} A((i-1, n))^{l_{i}}$, then

$$
\left\{\begin{array}{l}
d_{\bar{q}}(f(0)) \leqq d_{\bar{q}}\left(\widetilde{\psi}_{j}^{\alpha}(0)\right)+o\left(g_{j}^{\alpha}(0)\right) \\
d_{\bar{q}}(f(0)) \leqq d_{\bar{q}}(h(0))
\end{array}\right.
$$


Proof. Put $S=\bigoplus_{\alpha=1}^{m-1} \bigoplus_{j=1}^{s_{\alpha}} \psi_{j}^{\alpha} A((\alpha-1, n))$ and $H=\bigoplus_{i=1}^{m} A((i-1, n))^{l_{i}}$, then $A((0, n))^{2}=S \oplus H$ by Proposition 1.2.1). Consider the commutative diagrame

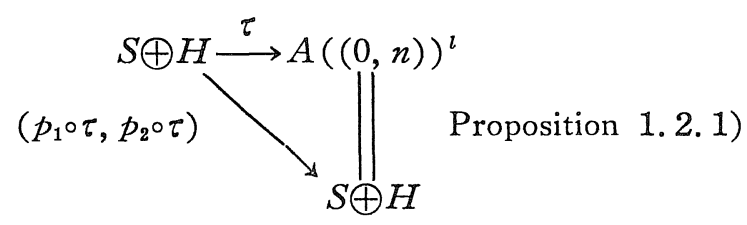

where $\tau$ is defined by $\tau\left(\sum \psi_{j}^{\alpha} g_{j}^{\alpha}+h\right)=\sum \widetilde{\psi}_{j}^{\alpha} g_{j}^{\alpha}+h$ and $p_{1}, p_{2}$ are the projections to $S, H$ respectively. Since $\widetilde{\psi}_{j}^{\alpha}-\psi_{j}^{\alpha} \in \mathfrak{\pi} A((0, n)) i d_{S}-\left.p_{1} \circ \tau\right|_{S}$ maps $\mathfrak{n}^{r} S$ to $\mathfrak{n}^{r+1} S$ for any integer $r \geqq 0$, so that we can define $i d_{S}+\sum_{i=1}^{\infty} \lambda^{i}$ with $\lambda=i d_{S}-\left.p_{1} \circ \tau\right|_{S}$ on $S$. We define $\kappa: S \oplus H \rightarrow S \oplus H$ to be the map

$$
\kappa(a, b)=\left(\left(i d_{S}+\sum_{i=1}^{\infty} \lambda^{i}\right)(a), b-p_{2} \circ \tau \circ\left(i d_{S}+\sum_{i=1}^{\infty} \lambda^{i}\right)(a)\right) \text {. }
$$

Then it is easily seen that

$$
\kappa \circ\left(p_{1} \circ \tau, p_{2} \circ \tau\right)=i d_{s \oplus H}, \quad\left(p_{1} \circ \tau, \quad p_{2} \circ \tau\right) \circ \kappa=i d_{S \oplus H} .
$$

Hence $\left(p_{1} \circ \tau, p_{2} \circ \tau\right)$ is an isomorphism, and so is $\tau$. This proves 1). 2) is clear by Proposition 1.2.2).

Q.E.D.

Theorem 1.6. Notations being as above, we have

$$
M_{1}=\bigoplus_{\alpha=1}^{m-1} \bigoplus_{j=1}^{s_{\alpha}} \widetilde{\psi}_{j}^{\alpha} A((\alpha-1, n)) \text {. }
$$

Proof. $\quad M_{1} \subset \bigoplus_{\alpha=1}^{m-1} \bigoplus_{j=1}^{S_{\alpha}} \widetilde{\psi}_{j}^{\alpha} A((\alpha-1, n))$ is clear by $(\stackrel{*}{*}), \quad\left(*_{*}^{*}\right)$, and $\left(\begin{array}{c}* * \\ * *\end{array}\right)$. So it is enough to show that $M_{1} \cap \bigoplus_{i=1}^{m} A((i-1, n))^{l_{i}}=0$. But this is just what the direct sum $\left(^{*}\right)$ means.

Q.E.D.

\section{§2. A Method to Compute a Free Resolution}

Let $I$ be an ideal in $R=k\left[\left[x_{1}, \cdots, x_{n}\right]\right]$. The aim of this section is to give an algorithm to compute a free resolution for $I$. We begin by summarizing the generalised Weierstrass preparation theorem. Let $\mathfrak{m}=\left(x_{1}, \cdots, x_{n}\right) R$ be the maximal ideal of $R$ and suppose depth $\mathfrak{m} R / I=d$. After a suitable linear coordinate transformation we may assume without 
loss of generality that $x_{n-d+1}, \cdots, x_{n}$ is a maximal $R / I$-regular sequence in $m$. Put $m=n-d$ and

$$
\text { (2.1.1) } \quad \bar{I}=\left\{\begin{array}{ll}
\bar{f} \in k\left[\left[x_{1}, \cdots, x_{m}\right]\right] & \begin{array}{l}
\bar{f}=f\left(\bmod \left(x_{m+1}, \cdots, x_{n}\right) R\right) \\
\text { for some } f \in I
\end{array}
\end{array}\right\} .
$$

Let $u_{i j}(1 \leqq i, j \leqq m)$ be indeterminates over $k\left[\left[x_{1}, \cdots, x_{m}\right]\right]$ and $K$ denote the field generated by $u_{i j}(1 \leqq i, j \leqq m)$ over $k$. Define $z=\left(z_{1}, \cdots, z_{m}\right)$ $\in K\left[\left[x_{1}, \cdots, x_{m}\right]\right]^{m}$ by the equations $x_{i}=\sum_{j=1}^{m} u_{j i} z_{j}(1 \leqq \mathrm{i} \leqq m) . \quad$ Then $\bar{I} K\left[\left[x_{1}, \cdots, x_{m}\right]\right]=\bar{I} K\left[\left[z_{1}, \cdots, z_{m}\right]\right]$ and $E(z ; \bar{I})$ is defined as a subset of $\boldsymbol{Z}_{0}^{m}$ by

(2.1.2) $\quad E(z ; \bar{I})=\left\{\operatorname{lex}_{z}\right.$ in $\left.(\bar{F}) \mid \bar{F} \in \bar{I} K\left[\left[z_{1}, \cdots, z_{m}\right]\right]\right\}$.

See $\left[9 ;\right.$ p. 280] for the definition of $\operatorname{lex}_{z} P$ where $P$ is a polynomial. $E(z ; \bar{I})$ has the following properties (see [8; Chap. 1]):

(2.1.3) There exists a Zariski open set $U$ in $G L(m, k)$ such that for every $a=\left(a_{i j}\right) \in U, E(z ; \bar{I})$ coincides with $\left\{\operatorname{lex}_{\left(y_{1}, \ldots, y_{m}\right)}\right.$ in $\left.(\bar{f}) \mid \bar{f} \in \bar{I}\right\}$, where $y_{1}, \cdots, y_{m} \in k\left[\left[x_{1}, \cdots, x_{m}\right]\right]$ are defined by the equations $x_{i}$ $=\sum_{j=1}^{m} a_{j i} y_{j} \quad(1 \leqq i \leqq m)$.

(2.1.4) $\quad E(z ; \bar{I})+Z_{0}^{m}=E(z ; \bar{I})$,

(2.1.5) $\quad\left(\nu_{1}, \cdots, \nu_{m}\right) \in E(z ; \bar{I})$ implies

$$
\left(\nu_{1}, \cdots, \nu_{i}, \sum_{j=i+1}^{m} \nu_{j}, 0, \cdots, 0\right) \in E(z ; \bar{I}) \quad \text { for } 1 \leqq i \leqq m-1 .
$$

Put $E=E(z ; \bar{I})$. The structure of $E$ is known in detail. Let us summarize the results we need later on.

First define $E_{i} \subset Z_{0}^{i}$ by $E_{i}=\left\{\alpha \in Z_{0}^{i} \mid(\alpha, 0, \cdots, 0) \in E\right\}$ for $1 \leqq i \leqq m$ and then define $\Gamma_{i}^{\prime}, \Gamma_{i}, \Delta_{i}$ for $1 \leqq i \leqq m-1$ inductively as follows (see [8; Chap. 1]) :

$$
\left\{\begin{array}{l}
\Gamma_{i}^{\prime}=\boldsymbol{Z}_{0}^{i} \backslash\left(E_{i} \cup \bigcup_{j=1}^{i-1} \Gamma_{j} \times \boldsymbol{Z}_{0}^{i-j}\right) \\
\Delta_{i}= \begin{cases}\alpha \in \Gamma_{i}^{\prime} & \begin{array}{l}
(\alpha, 0) \notin E_{i+1} \text { and there exists a positive } \\
\text { integer } d \text { such that }(\alpha, d) \in E_{i+1}
\end{array} \\
\Gamma_{i}=\Gamma_{i}^{\prime} \backslash \Delta_{i} .\end{cases}
\end{array}\right.
$$

We put $\Delta_{0}=\{\phi\}$ for convenience sake and further define

$$
\Gamma_{m}=\boldsymbol{Z}_{0}^{m} \backslash\left(E \cup \bigcup_{j=1}^{m-1} \Gamma_{j} \times \boldsymbol{Z}_{0}^{m-j}\right) .
$$


For each $\delta \in \Delta_{i}$ let $d(\delta)$ be the minimum of $d$ such that $(\delta, d) \in E_{i+1}$, in particular $d(\phi)$ is the smallest number of $E_{1}$. And put $A_{i \delta}=(\delta, d(\delta)$, $0, \cdots, 0)$. Then we have the following properties:

$$
\left\{\begin{array}{c}
\mathbb{Z}_{0}^{m}=\bigcup_{i=0}^{m-1} \bigcup_{\delta \in \Delta_{i}}\left(A_{i \delta}+\mathbb{Z}_{0}(i)\right) \cup \bigcup_{j=1}^{m} \Gamma_{j} \times \mathbb{Z}_{0}^{m-j} \\
\text { (disjoint union) } \\
E=\bigcup_{i=0}^{m-1} \bigcup_{\delta \in \Delta_{i}}\left(A_{i \delta}+\mathbb{Z}_{0}(i)\right),
\end{array}\right.
$$

where $\mathbb{Z}_{0}(i)=\left\{\alpha=\left(\alpha_{1}, \cdots, \alpha_{m}\right) \in \mathbb{Z}_{0}^{m} \mid \alpha_{1}=\cdots=\alpha_{i}=0\right\}$.

(2.1.7) $\bigcup_{\delta \in \Delta_{i-1}} \delta \times[0, d(\delta))=\Delta_{i} \cup \Gamma_{i} \quad$ (disjoint) for $1 \leqq i \leqq m$ and $\Delta_{m}$ $=e m p t y$.

(2.1.8) If $\left(\nu_{1}, \cdots, \nu_{i}\right) \in \Delta_{i}$ then $\left(\nu_{1}, \cdots, \nu_{i_{0}}\right) \in \Delta_{i_{0}}$ for any $i_{0}<i$.

The property (2.1.3) allows us to assume that $\left\{\operatorname{lex}_{\left(x_{1}, \ldots, x_{m}\right)}\right.$ in $(\bar{f})$ $\mid \bar{f} \in \bar{I}\}$ coincides with $E(z ; \bar{I})$, so we shall continue the description with this assumption from now on.

Remark 2.2. Denote $\bigoplus_{j=1}^{m} \bigoplus_{r \in \Gamma_{j}} x^{r} k((j, n))$ by $N_{E}$. We deduce from $(2.1 .6)$

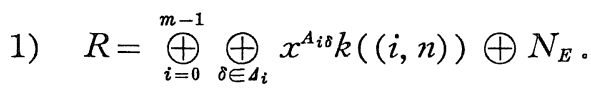

Let $x_{t}^{\nu_{t}} \cdots x_{n}^{\nu_{n}}$ be a monomial such that $\nu_{t} \neq 0$. For any monomial $x^{\alpha} \in R$ we can write uniquely

$$
x_{t}^{\nu_{t}} \cdots x_{n}^{\nu_{n}} x^{\alpha}=\sum_{i=0}^{m-1} \sum_{\delta \in \Delta_{i}} g_{i \delta} x^{A_{i \delta}}+r
$$

with $g_{i \delta} \in k((i, n))$ and $r \in N_{E}$ by 1$)$. If $x^{\alpha} \in N_{E}$ or $x^{\alpha}=x^{A_{g \varepsilon}}$ for some $t \leqq j \leqq m-1, \varepsilon \in \Delta_{j}$, then we have

2) $g_{i \delta}=0$ for $i \leqq t-2, \delta \in \Delta_{i}$

3) $\operatorname{deg}_{x_{t}} g_{t-1 \delta} \leqq \nu_{t}-1$ for $\delta \in \Delta_{t-1}$. In particular if $\nu_{t}=1, \operatorname{deg}_{x_{t}} g_{t-1 \delta}$ $=0$ i.e. $g_{t-1 \delta} \in k((t, n))$.

These follow immediately from the definition of $\Delta_{i}$ and (2.1.8).

Put $A=k((m, n))$ and $\mathfrak{n}=\left(x_{n+1}, \cdots, x_{n}\right) A$. Then $R=A((0, m))$. 
Theorem 2.3. (H. Grauert [5], H. Hironaka [7], [3]). There exists $f_{i \delta} \in I$ such that $f_{i \delta}-x^{A_{i \delta}} \in N_{E}$ and $o\left(\left(f_{i \delta}(0)\right)=o\left(x^{A_{i \delta}}\right)\right.$ for each $0 \leqq i \leqq m-1, \delta \in \Delta_{i}$, and we have the following:

1) $R=I \oplus N_{E}$

2) $\quad I=\bigoplus_{i=0}^{m-1} \bigoplus_{\delta \in \Delta_{i}} f_{i \delta} A((i, m))$

3) If $f=\sum_{i=0}^{m-1} \sum_{\delta \in \Delta_{i}} g_{i \delta} f_{i \delta}+r$ with $g_{i \delta} \in A((i, m))$ and $r \in N_{E}$, then $\left\{\begin{array}{l}o(f(0)) \leqq o\left(f_{i \delta}(0)\right)+o\left(g_{i \delta}(0)\right) \quad \text { for } 0 \leqq i \leqq m-1, \delta \in \Delta_{i} \\ o(f(0)) \leqq o(r(0)) .\end{array}\right.$

4) If $x_{\iota} f_{j \varepsilon}=\sum_{i=0}^{m-1} \sum_{\delta \in \Delta_{i}} g_{i \delta} f_{i \delta}+r$ with $g_{i \delta} \in A((i, m))$ and $r \in N_{E}$, then $r=0, g_{i \delta}=0$ for $i \leqq t-2, \delta \in \Delta_{i}$, and $g_{t-1 \delta} \in A((t, m))$ for $\delta \in \Delta_{t-1}$, provided $t \leqq j, \varepsilon \in \Delta_{j}$.

5) If for $f \in N_{E} \quad x_{t} f=\sum_{i=0}^{m-1} \sum_{\delta \in \Delta_{i}} g_{i \delta} f_{i \delta}+r$ with $g_{i \delta} \in A((i, m))$ and $r \in N_{E}$, then $g_{i \delta}=0$ for $i \leqq t-2, \delta \in \Delta_{i}$, and $g_{t-1 \delta} \in A((t, m))$ for $\delta \in$ $A_{t-1}$.

Proof. Note first that $R / I$ is flat over $A$. Then the method of the proof of $[4$; Chap. $1(1.2 .7),(1.2 .8)]$ is also applicable to our case, in which we do not have to care convergence, and we get 1), 2) and 3). Compare the argument of (1.5). 4),5) follow easily from Remark 2.2 and the "division algorithm" since $f_{i \delta}-x^{A_{i \hat{o}}} \in N_{E}$. Q.E.D.

Corollary 2. 4. Under the conditions of Theorem 2.3 $\Delta_{0}, \Delta_{1}, \cdots$, $\Delta_{m-1}$ are not empty.

Proof. If $\Delta_{i}$ were empty for some $0 \leqq i \leqq m-1$ then we would have $\Gamma_{i+1}=\Gamma_{i+2}=\cdots=\Gamma_{m}=\phi$ by $(2.1 .3)$. But then Theorem 2.3.1) would imply

$$
R / I=N_{E}=\bigoplus_{j=1}^{i} \underset{r \in \Gamma_{j}}{\oplus_{j}} x^{r} A((j, m))
$$

which means depth $_{\mathfrak{m}} R / I \geqq d+1$. This contradicts the assumption that depth $_{\mathfrak{m}} R / I=d$.

Q.E.D.

Corollary 2.5. Under the conditions of Theorem 2.3 R/I is 
Cohen-Macaulay if and only if $\Gamma_{1}=\cdots=\Gamma_{m-1}=\phi$.

Proof: Easy and left to the reader.

Let $l_{i}(1 \leqq i \leqq m)$ be the number of elements of $\Delta_{i-1}$, and we set $l=\sum_{i=1}^{m} l_{i}, s_{\alpha}=\sum_{i=\alpha+1}^{m} l_{i}(0 \leqq \alpha \leqq m-1)$. For each $1 \leqq i \leqq m$ put $f_{i-1 \delta}\left(\delta \in \Delta_{i-1}\right)$ in a suitable order and write them, say, $f_{l-s_{i-1}+1}, f_{l-s_{i-1}+2}, \cdots, f_{l-s_{i}}$. Then Theorem 2.3.2) becomes

$$
I=\bigoplus_{i=1}^{m} \bigoplus_{j=1}^{l_{i}} f_{l-s_{i-1}+j} A((i-1, m)) .
$$

We can compute

$$
M_{1}=\left\{\psi={ }^{t}\left(\psi_{1}, \cdots, \psi_{l}\right) \in R^{l}=A((0, m))^{l} \mid \sum_{i=1}^{l} \psi_{i} f_{i}=0\right\}
$$

by Theorem 1.6. Let $\widetilde{\psi}_{j}^{\alpha}$, $\tilde{\chi}_{j}^{a}\left(1 \leqq \alpha \leqq m-1,1 \leqq j \leqq s_{\alpha}\right)$ be defined as in Section $1\left(*_{*}^{*}\right),\left(*_{*}^{*}\right)$, and $\left(*_{*}^{*}\right)$, then we have

$(\mathrm{I}-0) \quad \tilde{\chi}_{j}^{\alpha}(0) \in \bigoplus_{i=1}^{m} k((i-1, \mathrm{~m}))^{l_{i}}$,

(II-0) $\quad d_{\bar{q}}\left(\tilde{\chi}_{j}^{\alpha}(0)\right) \geqq 1+q_{l-s_{\alpha}+j} \quad$ for $\quad 1 \leqq \alpha \leqq m-1, \quad 1 \leqq j \leqq s$ where $q_{i}=o\left(f_{i}(0)\right)$ and $\bar{q}=\left(q_{1}, \cdots, q_{l}\right)$.

(I-0) is trivial and (II-0) is deduced from the defining equations (*) ) and Theorem 2.3.3). Hence we get by Theorem 1.6

$\left(2.6 . M_{1}\right) *$

$$
M_{1}=\bigoplus_{\alpha=1}^{m-1} \bigoplus_{j=1}^{s_{a}} \widetilde{\psi}_{j}^{\alpha} A((\alpha-1, m)) \text {. }
$$

Put $\quad l_{i}^{\prime}=s_{i} \quad(1 \leqq i \leqq m-1), \quad m^{\prime}=m-1, \quad s_{\alpha}^{\prime}=\sum_{i=\alpha+1}^{m^{\prime}} l_{i}^{\prime} \quad\left(0 \leqq \alpha \leqq m^{\prime}-1\right)$, $l^{\prime}=\sum_{i=1}^{m^{\prime}} l_{i}^{\prime}$ and $A^{\prime}=k\left(\left(m^{\prime}, n\right)\right)$. We set $f_{l^{\prime}-s_{a-1}+j}^{\prime}=\widetilde{\psi}_{j}^{a}$ for $1 \leqq \alpha \leqq m^{\prime}$, $1 \leqq j \leqq l_{\alpha}^{\prime}$, then $\left(2.6 . M_{1}\right) *$ becomes

(2.6. $\left.M_{1}\right)$

$$
M_{1}=\bigoplus_{i=1}^{m^{\prime}} \bigoplus_{j=1}^{l_{i^{\prime}}} f^{\prime}{ }_{l^{\prime}-s_{i-1}+j} A^{\prime}\left(\left(i-1, m^{\prime}\right)\right) .
$$

Thus we are in the same situation as before. Let

$$
M_{2}=\left\{\psi={ }^{t}\left(\psi_{1}, \cdots, \psi_{1}^{\prime}\right) \in R^{\prime}=A^{\prime}\left(\left(0, m^{\prime}\right)\right)^{\prime} \mid \sum_{i=-1}^{l^{\prime}} \psi_{i_{i}} f_{i}^{\prime}=-0\right\} .
$$

If $m^{\prime}=1$ then $M_{1}$ is a free $R$-module and $M_{2}=0$. If $m^{\prime} \geqq 2$ then we 
can compute $M_{2}$ defining $\widetilde{\psi}_{j}^{\prime \alpha}, \quad \tilde{\chi}_{j}^{\prime \alpha}\left(1 \leqq \alpha \leqq m^{\prime}-1,1 \leqq j \leqq s_{\alpha}^{\prime}\right)$ by the formulae $(\stackrel{*}{*}),\left(\begin{array}{l}* * \\ *\end{array}\right)$, and $\left(\begin{array}{l}* * \\ *\end{array}\right)$ of Section 1 using (2.6. $\left.M_{1}\right)$, and obtain $\left(2.6 . M_{2}\right) *$

$$
M_{2}=\bigoplus_{\alpha=1}^{m^{\prime}-1} \bigoplus_{j=1}^{s_{\alpha^{\prime}}} \widetilde{\psi}_{j}^{\prime \alpha} A^{\prime}\left(\left(\alpha-1, m^{\prime}\right)\right) .
$$

Note that in this case the condition corresponding to (II-0) above, namely

$$
\begin{array}{cl}
(\mathrm{II}-0)^{\prime} & d_{\bar{q}^{\prime}}\left(\tilde{\chi}_{j}^{\prime \alpha}(0)\right) \geqq 1+q_{l^{\prime}-s_{\alpha^{\prime}+j}}^{\prime} \text { for } 1 \leqq \alpha \leqq m^{\prime}-1,1 \leqq j \leqq s_{\alpha}^{\prime} \text { where } \\
& q_{i}^{\prime}=d_{\bar{q}}\left(f_{i}^{\prime}(0)\right) \text { and } \bar{q}^{\prime}=\left(q_{1}^{\prime}, \cdots, q_{l^{\prime}}^{\prime}\right)
\end{array}
$$

is deduced from Corollary 1.5.2). Continuing this procedure we can compute a free resolution for $R / I$ of length $m=n-\operatorname{depth}_{\mathfrak{m}} R / I$ on and on.

Example 2.7. When $n$ - $\operatorname{depth}_{\mathfrak{m}} R / I=2$ the results of this section appear essentially in [2]. If, in this case, $I$ is generated by homogeneous polynomials and $R / I$ is Cohen-Macaulay, then Theorem 2.3.2) becomes

$$
I=f_{1} k((0, n)) \oplus \bigoplus_{i=1}^{l_{2}} f_{1+i} k((1, n))
$$

where $f_{i}\left(1 \leqq i \leqq 1+l_{2}\right)$ are homogeneous polynomials in $I$ such that $\operatorname{deg} f_{1} \leqq \operatorname{deg} f_{1+i}$ for $1 \leqq i \leqq l_{2}$ and $l_{2}=\operatorname{deg} f_{1}$. We may assume without loss of generality that $\operatorname{deg} f_{i} \leqq \operatorname{deg} f_{i+1}$ for $2 \leqq i \leqq l_{2}$. The sequence of integers $\left(\nu_{l_{2}}, \nu_{l_{2}-1}, \cdots, \nu_{1}\right)$ with $\nu_{i}=\operatorname{deg} f_{1+i}\left(1 \leqq i \leqq l_{2}\right)$ is the "caractère numérique" appeared in [6].

Example 2.8. When $n-\operatorname{depth}_{\mathfrak{m}} R / I=3, R / I$ has a free resolution

$$
0 \longrightarrow R^{l_{3}} \stackrel{\lambda_{3}}{\longrightarrow} R^{l_{3}+2 l_{3}} \stackrel{\lambda_{2}}{\longrightarrow} R^{1+l_{2}+l_{3}} \stackrel{\lambda_{1}}{\longrightarrow} R \stackrel{\lambda_{0}}{\longrightarrow} R / I \longrightarrow 0
$$

where the matrices $\lambda_{1}, \lambda_{2}, \lambda_{3}$ enjoy the properties:

1) $\lambda_{1}=\left(f_{1}, f_{2}, \cdots, f_{l_{2}+1}, f_{l_{2}+2}, \cdots, f_{l_{2}+l_{3}+1}\right)$

2)

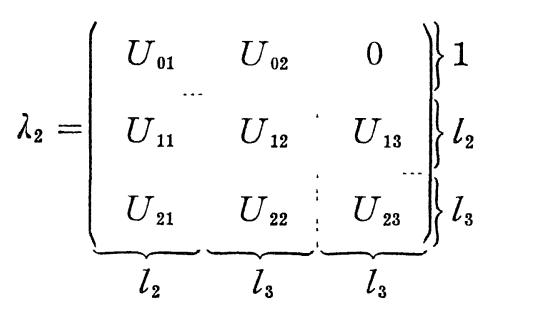


i) Each entry of $U_{01}, U_{02}, U_{12}$, and $U_{11}-x_{1} \cdot 1_{l_{2}}$ is in $k((1, n))$.

ii) Each entry of $U_{21}, \quad U_{13}, \quad U_{22}-X_{1} \cdot 1_{l_{3}}$, and $U_{23}-x_{2} \cdot 1_{l_{3}}$ is in $k((2, n))$.

3)

$$
\lambda_{3}=\left(\begin{array}{r}
-U_{13} \\
-U_{23} \\
U_{22}
\end{array}\right) \text { and } \lambda_{2} \cdot \lambda_{3}=0
$$

1) and 2) follow directly from the argument of this section while 3) holds by exactly the same reason as that of Corollaries 3.5.3)-3.5.4). Observe that one does not have to do any further computation to determine $\lambda_{3}$ if $\lambda_{2}$ is already known.

\section{§3. Main Results}

In this section we present a method to handle the ideal defining the minimal cone of a curve in $\mathbb{P}_{k}^{3}$ as an application of the results of the previous sections. As in the introduction 'curve' means an equidimensional complete scheme over a field $k$ of dimension 1 . We state the results in a slightly general situation which includes the case of our interest. Let $x_{1}, \cdots, x_{n}$ be indeterminates, $R=k\left[x_{1}, \cdots, x_{n}\right]$, and $\mathfrak{m}=\left(x_{1}\right.$, $\left.\cdots, x_{n}\right) R$. For any matrix $\phi$ with entries in $R$ we define $I(\phi)$ to be the ideal generated by $s \times s$ minors of $\phi$ where $s$ is the rank of $\phi$ (see $[1])$.

Proposition 3.1. Let $I$ be a homogeneous ideal in $R$ such that $\operatorname{dim} R / I \leqq n-2$ and $\operatorname{depth} \mathfrak{m} R / I \geqq n-3$, and let $J$ be any homogeneous subideal of $I$ such that $\operatorname{dim} R / J=\operatorname{depth}_{\mathfrak{m}} R / J=n-2$. Then, for a suitable choice of homogeneous coordinates, there exist homogeneous polynomials $f_{0}, f_{1}, \cdots, f_{a} \in J\left(\mathrm{a}=\operatorname{deg} f_{0}\right)$ and $f_{a+1}, \cdots, f_{a+b} \in I(b \geqq 0)$ such that

1) $J=f_{0} k(0, n) \oplus \bigoplus_{i=1}^{a} f_{i} k(1, n)$,

$$
I=\int_{0} k(0, n) \oplus \bigoplus_{i=1}^{a} f_{i} k(1, n) \oplus \bigoplus_{i=1}^{b} f_{a+i} k(2, n) .
$$

2) if for $1 \leqq j \leqq a+b, x_{1} f_{j}=\sum_{i=0}^{u+b} g_{i} f_{i}$ with

$$
{ }^{t}\left(g_{0}, \cdots, g_{a+b}\right) \in k(0, n) \oplus k(1, n)^{a} \oplus k(2, n)^{b} \text {, then } g_{0} \in k(1, n) \text {. }
$$


3) if for $a+1 \leqq j \leqq a+b, \quad x_{2} f_{j}=\sum_{i=0}^{a+b} g_{i} f_{i}{ }^{t}\left(g_{0}, \cdots, g_{a+b}\right) \in$

$$
\begin{aligned}
& k(0, n) \oplus k(1, n)^{a} \oplus k(2, n)^{b} \text {, then } g_{0}=0 \text { and } g_{i} \in k(2, n) \quad(1 \leqq i \\
& \leqq a+b) \text {. }
\end{aligned}
$$

Before proving the proposition we make a remark.

Remark 3.2. In Example 2.8 it is not always true that $U_{21}=0$. This implies that $f_{1} k((0, n)) \oplus\left(\bigoplus_{i=1}^{l_{2}} f_{1+i} k((1, n))\right.$ is not always an ideal of $R$. Thus Proposition 3.1 is somewhat different from Example 2.8.

Proof of Proposition 3.1. Let $R^{*}, I^{*}$ and $J^{*}$ be the m-adic completion of $R, I$ and $J$, respectively. After a suitable linear coordinate transformation we may assume that $x_{4}, \cdots, x_{n}$ (resp. $x_{3}, x_{4}, \cdots, x_{n}$ ) is an $R^{*} / I^{*}$-regular sequence (resp. a maximal $R^{*} / J^{*}$-regular sequence) in int. Put $\bar{R}^{*}=k((0,3)), \bar{I}^{*}=I^{*}\left(\bmod \left(x_{4}, \cdots, x_{n}\right) R^{*}\right)$, and $\bar{J}^{*}=J^{*}(\bmod$ $\left.\left(x_{4}, \cdots, x_{n}\right) R^{*}\right)$. Then $x_{3}$ becomes a maximal $R^{*} / J^{*}$-regular sequence. So we deduce from Theorem 2.3 that there exist homogeneous polynomials $\bar{f}_{0}, \cdots, \bar{f}_{a}\left(a=\operatorname{deg} \bar{f}_{0}\right.$, see Example 2.7 also $)$ such that

$$
\begin{aligned}
& \bar{R}^{*}=\bar{J}^{*} \oplus \bigoplus_{r \in \Gamma_{2}} x^{r} k((2,3)), \\
& \bar{J}^{*}=\bar{f}_{0} k((0,3)) \oplus \bigoplus_{i=1}^{a} \bar{f}_{i} k((1,3)) .
\end{aligned}
$$

We see from (1) that $\bar{I}^{*} / \bar{J}^{*} \cong \bar{I}^{*} \cap \underset{r \in \Gamma_{\mathbf{2}}}{\bigoplus} x^{r} k((2,3))$ is a $k\left[\left[x_{3}\right]\right]$ submodule of $\underset{r \in \Gamma_{2}}{\oplus} x^{r} k((2,3))=\underset{r \in \Gamma_{2}}{\bigoplus} x^{r} k\left[\left[x_{3}\right]\right]$, so that there exist homogeneous polynomials $\bar{f}_{a+1}, \cdots, \bar{f}_{a+b} \in \bar{I}^{*} \cap \underset{r \in \Gamma_{2}}{\oplus} x^{r} k((2,3))$ such that

$$
\bar{I}^{*} \cap \bigoplus_{r \in \Gamma_{2}} x^{r} k((2,3))=\bigoplus_{i=1}^{b} \bar{f}_{a+i} k\left[\left[x_{3}\right]\right]
$$

by elementary linear algebra over the principal ideal domain $k\left[\left[x_{3}\right]\right]$. Further there exist a subset $\Gamma \subset \Gamma_{2}$ and a nonnegative integer $e(\gamma)$ for each $r \in \Gamma$ such that

$$
\begin{aligned}
& \left.\bigoplus_{r \in \Gamma_{2}} x^{r} k\left[\left[x_{3}\right]\right]=\left\{I^{*} \cap \underset{\gamma \in \Gamma_{2}}{\oplus} x^{r} k\left[\left[x_{3}\right]\right]\right\} \underset{r \in \Gamma}{\oplus} \underset{0 \leqq i<e(r)}{\bigoplus} x^{r} x_{3}^{i} \cdot k\right\} \\
& \oplus\left\{\underset{r \in \Gamma_{2} \backslash \Gamma}{\oplus} x^{r} k\left[\left[x_{3}\right]\right]\right\} \text {. }
\end{aligned}
$$


It follows from (1), (2), (3), and (4) that

$$
\left\{\begin{array}{l}
\bar{I}^{*}=\bar{f}_{0} k((0,3)) \oplus \bigoplus_{i=1}^{a} \bar{f}_{i} k((1,3)) \oplus \underset{i=1}{\oplus} \bar{f}_{a+i} k((2,3)), \\
\bar{R}^{*}=\bar{I}^{*} \oplus\left\{\bigoplus_{r \in \Gamma} \bigoplus_{0 \leqq j<e(\gamma)} x^{\tau} x_{3}^{j} k \bigoplus_{\gamma \in \Gamma_{2} \backslash \Gamma} x^{\tau} k((2,3))\right\} .
\end{array}\right.
$$

Put $A^{*}=k((3, n))$. Let $f_{a+i}^{\prime}(1 \leqq i \leqq b)$ be homogeneous polynomials of $I^{*}$ such that $f_{a+i}^{\prime}(0)=\bar{f}_{a+i}$, and let $f_{i}(0 \leqq i \leqq a)$ be those homogeneous polynomials of $J^{*}$ described in (2.3). Then $f_{i}(0)=\bar{f}_{i}$ $(0 \leqq i \leqq a)$ and

$$
\left\{\begin{array}{l}
J^{*}=f_{0} A^{*}((0,3)) \oplus \underset{i=1}{a} \int_{i} A^{*}((1,3)), \\
R^{*}=J^{*} \oplus \underset{r \in \Gamma_{2}}{\bigoplus_{2}} x^{r} A^{*}((2,3)) .
\end{array}\right.
$$

Using (5) and noting that $R^{*} / I^{*}$ is flat over $A^{*}$ we deduce

$$
\left\{\begin{array}{l}
I^{*}=f_{0} A^{*}((0,3)) \oplus \bigoplus_{i=1}^{a} f_{i} A^{*}((1,3)) \oplus \bigoplus_{i=1}^{b} f_{a+i}^{\prime} A^{*}((2,3)), \\
R^{*}=I^{*} \oplus N^{*}
\end{array}\right.
$$

where $\quad N^{*}=\bigoplus_{r \in \Gamma^{r}} \underset{0 \leqq j<e(r)}{\bigoplus_{0}} x^{r} x_{3}^{j} A^{*} \oplus \underset{r \in \Gamma_{2} \backslash \Gamma^{r}}{\oplus} x^{r} A^{*}((2,3))$.

See the proof of Corollary 1.5 and $[4 ;(1,2,8)]$.

(7) enables us to find homogeneous polynomials $\tilde{f}_{a+i}$ in $N^{*}(1 \leqq i$ $\leqq b)$ such that $\tilde{f}_{a+i} \equiv f_{a+i}^{\prime}-f_{a \rightarrow i}^{\prime}(0)\left(\bmod I^{*}\right)$. Put $f_{a+i}=f_{a+i}^{\prime}(0)+\bar{f}_{a+i}$ $(1 \leqq i \leqq b)$, then $f_{a+i} \in I^{*} \cap \bigoplus_{r \in \Gamma_{2}} x^{r} A^{*}((2,3))$, and we again get (7) with $\left(f_{a+1}^{\prime}, \cdots, f_{a+b}^{\prime}\right)$ replaced by $\left(f_{a+1}, \cdots, f_{a+b}\right)$ since $f_{a+i}(0)=f_{a+i}^{\prime}(0)=\bar{f}_{a+i}$ for $1 \leqq i \leqq b . \quad I$ and $J$ being homogeneous this proves 1). 2) and 3) follow from Theorem 2.3.4)-2.3.5), and from the fact that $f_{a+i} \in$ $\bigoplus_{r \in \Gamma_{2}} x^{r} A^{*}((2,3))$ for $1 \leqq i \leqq b$.

Corollary 3.3. In Proposition 3.1

1) $0 \leqq b \leqq \sum_{i=1}^{a}\left(\operatorname{deg} f_{i}+i-a\right)$.

2) $\operatorname{dim} R / I=\left\{\begin{array}{lll}n-2 & \text { if } & b<\sum_{i=1}^{a}\left(\operatorname{deg} f_{i}+i-a\right), \\ n-3 & \text { if } & b=\sum_{i=1}^{a}\left(\operatorname{deg} f_{i}+i-a\right)\end{array}\right.$

Proof. Let $F(\nu)$ be the Hilbert function of $R / 1$. One can compute 
$F(\nu)$ using Proposition 3.1.1) and get

(1) $F(\nu)=\left(\begin{array}{c}n-1+\nu \\ n-1\end{array}\right)-\left(\begin{array}{c}n-1+\nu-a \\ n-1\end{array}\right)-\sum_{i=1}^{a}\left(\begin{array}{c}n-2+\nu-\operatorname{deg} f_{i} \\ n-2\end{array}\right)$

$$
-\sum_{i=1}^{b}\left(\begin{array}{c}
n-3+\nu-\operatorname{deg} f_{a+i} \\
n-3
\end{array}\right)
$$

for $\nu \gg 0$.

We deduce from (1)

$$
\begin{aligned}
F(\nu)= & \frac{1}{(n-3) !}\left\{\sum_{i=1}^{a}\left(\operatorname{deg} f_{i}+i-a\right)-b\right\} \nu^{n-3} \\
& +(\text { terms of degree }<n-3)
\end{aligned}
$$

for $\nu \gg 0$. Hence 1) follows. 2) is obvious since $\operatorname{dim} R / I \geqq \operatorname{depth}_{\mathfrak{m}} R / I$ $\geqq n-3$ by hypothesis.

Q.E.D.

In the situation of Proposition 3.1 we set $\nu_{j}=\operatorname{deg} f_{j}(0 \leqq j \leqq a+b)$, $\mu_{i j}=\nu_{j}+1-\nu_{i}(0 \leqq i \leqq a+b, 1 \leqq j \leqq a+b)$, and $\mu_{i, a+b+j}=\mu_{i, a+j}(1 \leqq j \leqq b)$. Then $\nu_{j}, \mu_{i j}$ enjoy the properties:

$$
\begin{cases}\text { 1) } & \mu_{i_{1} j_{1}}-\mu_{i_{2} j_{1}}=\mu_{i_{1} j_{2}}-\mu_{i_{2} j_{2}} \\ & \text { for } 0 \leqq i_{1}, i_{2} \leqq a+b, 1 \leqq j_{1}, j_{2} \leqq a+2 b \\ 2) & \mu_{i i}=1, \text { for } 0 \leqq i \leqq a+b . \\ 3) & \mu_{i, j+a+b}=\mu_{i, a+j}, \text { for } 1 \leqq j \leqq b . \\ 4) & \nu_{j}=\sum_{i=j+1}^{a} \mu_{i i}+\sum_{i=1}^{j-1} \mu_{i, i+1}, \text { for } 0 \leqq j \leqq a\end{cases}
$$

Corollary 3.5. In the situation of Proposition $3.1 \mathrm{R} / \mathrm{I}$ has a free resolution

$$
0 \longrightarrow R^{b} \stackrel{\lambda_{3}}{\longrightarrow} R^{a+2 b} \stackrel{\lambda_{2}}{\longrightarrow} R^{a+b+1} \stackrel{\lambda_{1}}{\longrightarrow} R \stackrel{\lambda_{0}}{\longrightarrow} R / I \longrightarrow 0
$$

such that the matrices $\lambda_{1}, \lambda_{2}, \lambda_{3}$ have the following properties:

1) $\lambda_{1}=\left(f_{0}, f_{1}, \cdots, f_{a}, f_{a+1}, \cdots, f_{a+b}\right)$.

2)

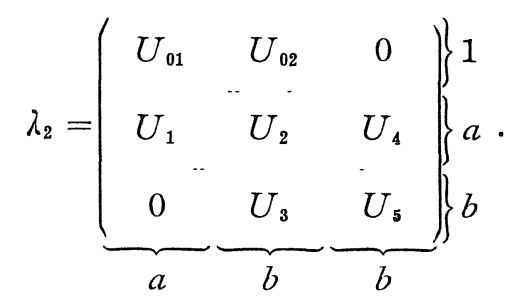


a) Each nonzero $(i, j)$ componenl of $\lambda_{2}$ is homogeneous of degree $\mu_{i j}$, reherc $0 \leqq i \leqq a+b, 1 \leqq i \leqq a+2 b$.

в) $U_{01}, U_{02}, U_{2}$, and $U_{1}-x_{1} \cdot 1_{a}$ take entries in $k(1, n)$.

r) $U_{4}, U_{3}-x_{1} \cdot 1_{b}$, and $U_{5}-x_{2} \cdot 1_{b}$ take entries in $k(2, n)$.

3)

$$
\lambda_{3}=\left(\begin{array}{r}
-U_{4} \\
-U_{5} \\
U_{3}
\end{array}\right) \text {. }
$$

4) $\lambda_{2} \cdot \lambda_{3}=0$.

5) $R / I\left(\begin{array}{l}U_{01} \\ U_{1}\end{array}\right)$ is a Cohen-Macaulay ring of dimension $n-2$, and $I\left(\lambda_{3}\right)$ contains an $R$-sequence of length 3 or $I\left(\lambda_{3}\right)=R$.

Proof. Let $\tilde{\chi}^{1}=\left(\tilde{\chi}_{i j}^{1}\right)$ be the matrix defined by the equations $x_{1} f_{j}$ $=\sum_{i=0}^{a+b} \tilde{\chi}_{i j}^{1} f_{i}$ with ${ }^{t}\left(\tilde{\chi}_{0 j}^{1}, \cdots, \tilde{\chi}_{a+b, j}^{1}\right) \in k(0, n) \oplus k(1, n)^{a} \oplus k(2, n)^{b}$ for $1 \leqq j \leqq a$ $+b$, and $\tilde{\chi}^{2}=\left(\tilde{\chi}_{i j}^{2}\right)$ the matrix defined by the equations $x_{2} f_{a+j}=\sum_{i=0}^{a+b} \tilde{\chi}_{i j}^{2} f_{i}$ with ${ }^{t}\left(\tilde{\chi}_{0 j}^{2}, \cdots, \tilde{\chi}_{a+b, j}^{2}\right) \in k(0, n) \oplus k(1, n)^{a} \oplus k(2, n)^{b}$ for $1 \leqq j \leqq b$. Put

$$
\begin{aligned}
& \left(\widetilde{\psi}_{i j}^{1}\right)=\left(\widetilde{\psi}_{1}^{1}, \cdots, \widetilde{\psi}_{a+b}^{1}\right)=\left(\begin{array}{l}
0 \cdots 0 \\
x_{1} 1_{a+b}
\end{array}\right)-\tilde{\chi}^{1}, \\
& \left(\widetilde{\psi}_{i j}^{2}\right)=\left(\widetilde{\psi}_{1}^{2}, \cdots, \widetilde{\psi}_{b}^{2}\right)=\left(\begin{array}{c}
0 \\
x_{2} 1_{b}
\end{array}\right)-\tilde{\chi}^{2},
\end{aligned}
$$

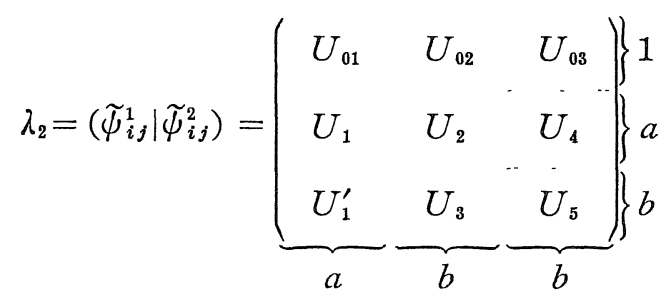

and $\lambda_{1}=\left(f_{0}, f_{1}, \cdots, f_{a+b}\right)$. Then $\tilde{\chi}_{i j}^{1}=0$ for $a+1 \leqq i \leqq a+b, 1 \leqq j \leqq a$ since $J=f_{0} k(0, n) \oplus \bigoplus_{i=1}^{a} f_{i} k(1, n)$ is an ideal of $R$. This implies $U_{1}^{\prime}=0 . \quad U_{03}=0$ by Proposition 3.1.3). 2. $\beta$ ), 2. $\gamma$ ) follow from 2) and 3) of Proposition 3.1. 2. $\alpha$ ) is obvious.

Now we verify by 2$)$ that $\tilde{\chi}^{\alpha}(\alpha=1,2)$ satisfy conditions (I) and (II) of Section 1 with $\bar{q}=\left(\operatorname{deg} f_{0}, \cdots, \operatorname{deg} f_{a+b}\right)$. Hence we deduce from Proposition 3.1.1) and Theorem 1.6 that

$$
\operatorname{Ker} \lambda_{1}=\bigoplus_{i=1}^{a+b} \widetilde{\psi}_{i}^{1} k(0, n) \oplus \bigoplus_{i=1}^{b} \widetilde{\psi}_{i}^{2} k(1, n)=\operatorname{Im} \lambda_{2} .
$$


Let $\lambda_{3}$ be the matrix defined by the formula 3 ), and let $\widetilde{\phi}_{1}, \cdots, \widetilde{\phi}_{b}$ be its column vectors. We must show that $\operatorname{Ker} \lambda_{2}=\operatorname{Im} \lambda_{3}$. First observe that each column vector of $\lambda_{3}-\left(\begin{array}{c}0 \\ x_{1} 1_{b}\end{array}\right)$ is in $k(0, n)^{a+b} \oplus_{b} k(1, n)^{b}$, so that if we have $\lambda_{2} \cdot \lambda_{3}=0$, Ker $\lambda_{2}$ must be equal to $\operatorname{Im} \lambda_{3}=\bigoplus_{i=1}^{b} \widetilde{\phi}_{i} k(0, n)$ by Theorem 1.6. But it is easily seen that each column vector of $\lambda_{2} \cdot \lambda_{3}$ is in $k(0, n) \oplus k(1, n)^{a} \oplus k(1, n)^{b}$ by 2$)$ and that $\lambda_{1} \cdot\left(\lambda_{2} \cdot \lambda_{3}\right)=\left(\lambda_{1} \cdot \lambda_{2}\right) \cdot \lambda_{3}=0$. Hence $\lambda_{2} \cdot \lambda_{3}=0$ by Proposition 3.1.1), and $\operatorname{Ker} \lambda_{2}=\operatorname{Im} \lambda_{3}$. Thus (A) is exact.

$$
I\left(\begin{array}{l}
U_{01} \\
U_{1}
\end{array}\right)=J \text { since }
$$

$$
\left(\mathrm{A}_{J}\right) \quad 0 \longrightarrow R^{a} \stackrel{\left(\begin{array}{l}
U_{01} \\
U_{1}
\end{array}\right)}{\longrightarrow} R^{a+1} \stackrel{\left(f_{0}, \cdots, f_{a}\right)}{\longrightarrow} R \stackrel{\lambda_{0}}{\longrightarrow} R / J \longrightarrow 0
$$

is exact by (A) applied to $J$. So the first part of 5) follows. The last part of 5 ) is merely the criterion of [1; Corollary 1]. Q.E.D.

Corollary 3.6. In Corollary 3.5 we set

$$
W_{1}=\left(\begin{array}{ll}
U_{01} & U_{02} \\
U_{1} & U_{2} \\
0 & U_{3}
\end{array}\right), W_{2}=\left(\begin{array}{ll}
U_{01} & 0 \\
U_{1} & U_{4} \\
0 & U_{5}
\end{array}\right)
$$

and let $W_{i}^{(j)}(0 \leqq j \leqq a+b, i=1,2)$ denote the square matrix obtained by leaving out the $j$-th row from $W_{i}$. Then we have for some $\varepsilon(\neq 0) \in k$

1) $\left(\operatorname{det} U_{3}\right) f_{j}=(-1)^{j} \cdot \varepsilon \cdot \operatorname{det} W_{1}^{(j)}$ for $0 \leqq j \leqq a+b$,

2) $\quad\left(\operatorname{det} U_{5}\right) f_{j}=(-1)^{j} \cdot \varepsilon \cdot \operatorname{det} W_{2}^{(j)}$ for $0 \leqq j \leqq a+b$.

Proof. Put $G_{i}=\left(\operatorname{det} W_{i}^{(0)},-\operatorname{det} W_{i}^{(1)}, \cdots,(-1)^{a+b} \operatorname{det} W_{i}^{(a+b)}\right) \quad(i=1$, 2). Since $\lambda_{0} W_{i}=0, G_{i} W_{i}=0$, rank $W_{i}=a+b$ for $i=1,2$, and ht $I \geqq 2$ we find that $u_{i} \lambda_{0}=G_{i}$ for some $u_{i} \in R$, so that $u_{i} f_{0}=\operatorname{det} W_{i}^{(0)}=\operatorname{det} U_{1}$ - $\operatorname{det} U_{2 i+1}(i=1,2)$. But we know that $f_{0}=\varepsilon \cdot \operatorname{det} U_{1}$ for some $\varepsilon(\neq 0) \in k$, thus $\varepsilon u_{i}=\operatorname{det} U_{2 i+1}(i=1,2)$ which implies 1) and 2).

Q.E.D.

Next theorem is a converse version of Proposition 3.1 and Corollaries $3.3,3.5$ and 3.6 .

Theorem 3. 7. Lel $\mu_{i j}(0 \leqq i \leqq a+b, 1 \leqq j \leqq a+2 b), \nu_{j}$ be integers 


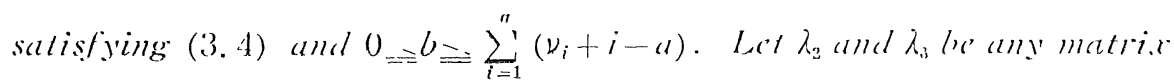
satisfying the conditions 2), 3), 4) and 5) of ('orollary 3.5 and set $W_{1}, W_{2}$ as in Corollary 3.6. Then we have

1) $\operatorname{det} W_{1}^{(j)}\left(\right.$ resp. $\left.\operatorname{det} W_{2}^{(j)}\right)$ is divisible by $\operatorname{det} U_{3}$ (resp. $\operatorname{det} U_{5}$ ).

2) Put $f_{j}=(-1)^{j} \operatorname{det} W_{1}^{(j)} / \operatorname{det} U_{3}$, and let $I$ (resp. $\left.J\right)$ be the homogeneous ideal in $R$ generated by $f_{0}, \cdots, f_{a \dashv b}$ (resp. $\left.f_{0}, \cdots, f_{n}\right)$, then

i) $J=f_{0} k(0, n) \oplus \underset{i=1}{a} f_{i} k(1, n)$,

ii) $\quad I=f_{0} k(0, n) \oplus \bigoplus_{i=1}^{a} f_{i} k(1, n) \oplus \bigoplus_{i=1}^{b} f_{a+i} k(2, n)$ and $R / I$ has $a$ free resolution of the form (A).

3) $\operatorname{dim} R / I \leqq n-2$ and

$$
\operatorname{depth}_{\mathfrak{m}} R / I=\left\{\begin{array}{lll}
n-2 & \text { if } & I\left(\lambda_{3}\right)=R \\
n-3 & \text { if } & I\left(\lambda_{3}\right) \neq R
\end{array}\right.
$$

Proof of 1). Note first that $\operatorname{det} W_{1}^{(j)}$ (resp. $\operatorname{det} W_{2}^{(j)}$ ) is evidently divisible by $\operatorname{det} U_{3}$ (resp. $\operatorname{det} U_{5}$ ) for $1 \leqq j \leqq a$. Put

$$
G_{i}=\left(\operatorname{det} W_{i}^{(0)},-\operatorname{det} W_{i}^{(1)}, \cdots,(-1)^{a+b} \operatorname{det} W_{i}^{(a+b)}\right)
$$

and $f_{j}=(-1)^{j} \operatorname{det}\left(\begin{array}{l}U_{10} \\ U_{1}\end{array}\right)^{(j)}$ for $0 \leqq j \leqq a$, where $\left(\begin{array}{l}U_{01} \\ U_{1}\end{array}\right)^{(j)}$ denotes the matrix obtained by leaving out the $j$-th row from $\left(\begin{array}{l}U_{01} \\ U_{1}\end{array}\right)$. Obviously

$$
\left\{\begin{array}{l}
\operatorname{det} W_{1}^{(j)}=(-1)^{j}\left(\operatorname{det} U_{3}\right) f_{j} \\
\operatorname{det} W_{2}^{(j)}=(-1)^{j}\left(\operatorname{det} U_{5}\right) f_{j}
\end{array}\right.
$$

for $0 \leqq j \leqq a$, and $f_{0}, \cdots, f_{a}$ have no common factor other than units by the condition 3.5.5). This enables us to write $G_{i}=h_{i} K_{i}(i=1,2)$, where $K_{i}$ ia a row vector in $R^{a+b+1}$ without any common factor except units among the entries, and $h_{i} \in R$ divides $\operatorname{det} U_{2 i+1}$ for $i=1,2$. Put $u_{i}=$ $\operatorname{det} U_{2 i+1} / h_{i}(i=1,2)$. Then, for $i=1,2, u_{i}$ is a homogenous polynomial of $k\left[x_{i}, x_{3}, x_{4}\right]$ which is monic in $x_{i}$. Observe that $K_{1}=\left(u_{1} f_{0},-u_{1} f_{1}, \cdots\right.$, $\left.(-1)^{a} u_{1} f_{a}, \cdots\right)$ and $K_{2}=\left(u_{2} f_{0},-u_{2} f_{1}, \cdots,(-1)^{a} u_{2} f_{a}, \cdots\right)$ by (1). We want to show that $h_{1}$ (resp. $h_{2}$ ) is in fact equal to $\operatorname{det} U_{3}$ (resp. $\operatorname{det} U_{3}$ ) 
up to units. It is enough to show that both $u_{1}$ and $u_{2}$ are units. The condition $\lambda_{2} \cdot \lambda_{3}=0$ can be expressed in the following form:

$$
\left(\begin{array}{l}
U_{02} \\
U_{2} \\
U_{3}
\end{array}\right) U_{5}=\left(\begin{array}{ll}
U_{01} & 0 \\
U_{1} & U_{4} \\
0 & U_{5}
\end{array}\right)\left(\begin{array}{r}
-U_{4} \\
U_{3}
\end{array}\right)
$$

$G_{2}\left(\begin{array}{c}U_{01} \\ U_{1} \\ 0\end{array}\right)=0$ and $G_{2}\left(\begin{array}{c}0 \\ U_{4} \\ U_{5}\end{array}\right)=0$ by the definition of $G_{2}$, so that we get $G_{2} W_{1}$ $=0$ by (2). On the other hand $G_{1} W_{1}=0$ by the definition of $G_{1}$, thus we obtain $K_{1} W_{1}=K_{2} W_{1}=0$. Since $\operatorname{det} W_{1}^{(0)}$ is a non-zero polynomial monic in $x_{1}, W_{1}$ has the maximal rank $a+b$. We have therefore that $A K_{1}=A K_{2}$ for some relatively prime polynomials $A, B \in R$. But $A$ and $B$ must be units, since the entries of $K_{i}$ have no common factor other than units for $i=1,2$. Thus $K_{1}=s K_{2}$ with $s \in k$, and hence $u_{1} f_{j}=s u_{2} f_{j}$ for $0 \leqq j \leqq a$. This implies $u_{1}=s u_{2}$, and we conclude that both $u_{1}$ and $u_{2}$ must be units, because $u_{i}$ is a homogeneous polynomial of $k\left[x_{i}, x_{3}, x_{4}\right]$ which is monic in $x_{i}$ for $i=1,2$.

Proof of 2). It is trivial that

$$
0 \longrightarrow R^{b} \stackrel{\lambda_{3}}{\longrightarrow} R^{a+2 b} \stackrel{\lambda_{2}}{\longrightarrow} R^{a+b+1} \stackrel{\lambda_{1}}{\longrightarrow} R \stackrel{\lambda_{0}}{\longrightarrow} R / I \longrightarrow 0
$$

is a complex. To prove exactness we need only verify the conditions of $[1$; Corollary 1]. The condition on ranks is obviously satisfied. Let $f, g$ be an $R$-sequence in $J$, and let $H$ be the ideal

$$
\left(f \cdot \operatorname{det} U_{3}, f \cdot \operatorname{det} U_{5}, g \cdot \operatorname{det} U_{3}, g \cdot \operatorname{det} U_{5}\right) R .
$$

Then the height of $H$ is equal to or larger than 2 since $\operatorname{det} U_{3}$ and $\operatorname{det} U_{5}$ are relatively prime. In addition, $H$ is contained in $I\left(\lambda_{2}\right)$, because both $f$ and $g$ are linear combinations of $f_{j}=(-1)^{j} \operatorname{det} W_{1}^{(j)} / \operatorname{det} U_{3}$ $=(-1)^{j} \operatorname{det} W_{2}^{(j)} / \operatorname{det} U_{5}(0 \leqq j \leqq a)$. Hence $I\left(\lambda_{2}\right)$ contains an $R$-sequence of length 2 . ht $I\left(\lambda_{2}\right) \geqq$ ht $J=2 \geqq 1$ and $I\left(\lambda_{3}\right)$ contains an $R$-sequence of length 3 or $I\left(\lambda_{3}\right)=R$ by assumption, thus the complex above is exact.

Set $\left(\widetilde{\phi}_{1}, \cdots, \widetilde{\phi}_{b}\right)=\lambda_{3}$ and $\left(\widetilde{\psi}_{1}, \cdots, \widetilde{\psi}_{a+2 b}\right)=\lambda_{2}$. We know by Corollary 1.5 that

$$
R^{a+2 b}=\bigoplus_{i=1}^{b} \widetilde{\phi}_{i} k(0, n) \oplus\left\{k(0, n)^{a+b} \oplus k(1, n)^{b}\right\},
$$




$$
\begin{aligned}
k^{a+b+1}= & \{\overbrace{i=1}^{a+b} \tilde{\psi}_{i} k(0, n) \oplus \underset{i=1}{\bigoplus_{i}} \bar{\psi}_{a+n+i} k(1, n)\} \\
& \oplus\left\{k(0, n) \oplus k(1, n)^{a} \oplus k(2, n)^{b}\right\} .
\end{aligned}
$$

We have $\widetilde{\phi}_{i} \in \operatorname{Im} \lambda_{3}=\operatorname{Ker} \lambda_{2}$ for $1 \leqq i \leqq b$, so that we deduce from (3) and (4) that

$$
\operatorname{Ker} \lambda_{1}=\operatorname{Im} \lambda_{2}=\bigoplus_{i=1}^{a+b} \widetilde{\psi}_{i} k(0, n) \oplus \bigoplus_{i=1}^{b} \widetilde{\psi}_{a+b+1} k(1, n)
$$

Using (4) and (5) we find that any element of $\operatorname{Im} \lambda_{1}$ can be written $\sum_{i=0}^{a+b} g_{i} f_{i}$ with ${ }^{t}\left(g_{0}, \cdots, g_{a+b}\right) \in k(0, n) \oplus k(1, n)^{a} \oplus k(2, n)^{b}$, and that $k(0, n)$ $\oplus k(1, n)^{a} \oplus k(2, n)^{b} \cap \operatorname{Ker} \lambda_{1}=0$. Thus we obtain

$$
\operatorname{Im} \lambda_{1}=f_{0} k(0, n) \oplus \underset{i=1}{a} f_{i} k(1, n) \oplus \underset{i=1}{b} f_{a+i} k(2, n)
$$

This proves 2-ii). 2-i) is proved similarly. 3) is obvious. Q.E.D.

Remark 3.8. In the case $n=4$, if one wishes to deal with the ideal in $R$ defining the minimal cone of a curve in $\mathbb{P}_{k}^{3}, b$ must be taken to be strictly smaller than $\sum_{i=1}^{a}\left(\nu_{i}+i-a\right)$ and the condition 5) of Corollary 3.5 should be altered as follows:

$(3.5 .5)^{\prime} \quad R / I\left(\begin{array}{l}U_{01} \\ U_{1}\end{array}\right)$ is a Cohen-Macaulay ring of dimension 2 and $I\left(\lambda_{3}\right)$ contains an $R$-sequence of length 4 or $I\left(\lambda_{3}\right)=R$.

Remark 3.9. The conclusions from Proposition 3.1 to Corollary 3.6 are also valid for any ideal $I^{*} \subset R^{*}=k\left[\left[x_{1}, \cdots, x_{n}\right]\right]$ such that depth $R^{*} / I^{*}$ $\geqq n-3$ and $\operatorname{dim} R^{*} / I^{*} \leqq n-2$.

\section{$\S 4$. Discussions in the Case $b=\mathbb{1}$}

In Theorem 3.7 the relation $\lambda_{2} \cdot \lambda_{3}=0$ is essential. When $b=1$ this relation is rather easy to solve provided that $n=4$ and $I\left(\lambda_{3}\right)$ contains an $R$-sequence of length 4 or $I\left(\lambda_{3}\right)=R$. The aim of this section is to illustrate how Theorem 3.7 works in this special case.

We assume the field $k$ to be algebraically closed with characteristic 
0 throughout this section. We begin with a remark.

Remark 4.1. Let $\lambda_{2}, \lambda_{3}$ be as in Corollary 3.5.2) and 3). Using Lemma 1.3 twice with $\alpha=1,2$ and $s_{1}=s_{2}=b$, we get

1) $\quad k(0, n)^{b}=k(0, n)^{b} U_{3} \oplus k(1, n)^{b} U_{5} \oplus k(2, n)^{b}$ where $k(i, n)^{b}(i=0,1,2)$ denote the sets of row vectors.

Set

2) $\left\{\begin{array}{l}U_{3}-x_{1} 1_{b}=-\stackrel{\circ}{U}_{3}, \quad U_{5}-x_{2} 1_{b}=-\stackrel{\circ}{U}_{5}, \\ \left(\begin{array}{l}U_{01} \\ U_{1}\end{array}\right)=\left(\begin{array}{c}0 \\ x_{1} 1_{a}\end{array}\right)+\sum_{r \geqq 0} x_{2}^{r} V^{(r)},\end{array}\right.$

where $V^{(r)}$ are matrices with entries in $k(2, n)$. Then we see by 1$)$ and 2) that $\lambda_{2} \cdot \lambda_{3}=0$ is equivalent to

3) $\left\{\begin{array}{l}\left(\begin{array}{l}0 \\ 1_{a}\end{array}\right) U_{4} \stackrel{\circ}{U}_{3}+\sum_{r \geq 0} V^{(r)} U_{4} \stackrel{\circ}{U}_{5}^{r}=0 \\ \stackrel{\circ}{U}_{3} \stackrel{\circ}{U}_{5}=\stackrel{\circ}{U}_{5} \stackrel{\circ}{U}_{3} \\ \left(\begin{array}{l}U_{02} \\ U_{2}\end{array}\right)=-\sum_{r \geq 1} \sum_{i=0}^{r-1} x_{2}^{r-i-1} V^{(r)} U_{4} \stackrel{\circ}{U}_{5}^{i}\end{array}\right.$

Now we restrict ourselves to the case where $n=4$ and $b=1$. Let $\Lambda_{2}$ be a matrix $\left(\mu_{i j}\right)$ satisfying (3.4), and let $S\left(\Lambda_{2}\right)$ be the set of subschemes of $\mathbf{P}_{k}^{3}$ defined by

$$
S\left(\Lambda_{2}\right)=\left\{\begin{array}{l|l}
\text { Proj } R / I & \begin{array}{l}
I \text { is defined as in 3.7.2) by } \\
\text { a matrix } \lambda_{2} \text { satisfying the } \\
\text { conditions of Theorem 3.7. }
\end{array}
\end{array}\right\} \text {. }
$$

Let $I(X)$ denote the ideal $f_{0} k(0,4) \oplus \bigoplus_{i=1}^{a} f_{i} k(1,4) \oplus f_{a+1} k(2,4)$ defining $X \in S\left(\Lambda_{2}\right)$. We may assume without loss of generality that $\nu_{0} \leqq \nu_{1} \leqq \cdots \leqq \nu_{a}$ (see Example 2.7). After the change of variables $\left(x_{1}-\stackrel{\circ}{U}_{3}, x_{2}-\stackrel{\circ}{U}_{5}, x_{3}\right.$, $\left.x_{4}\right) \rightarrow\left(x_{1}^{\prime}, x_{2}^{\prime}, x_{3}^{\prime}, x_{4}^{\prime}\right)$ we may assume that $\stackrel{\circ}{U}_{3}=\stackrel{\circ}{U}_{5}=0$. Then 4.1 .3$)$ becomes

$$
\left\{\begin{array}{l}
V^{(0)} U_{4}=0 \\
\left(\begin{array}{l}
U_{02} \\
U_{2}
\end{array}\right)=-\sum_{r \geq 1} x_{2}^{r-1} V^{(r)} U_{4}
\end{array}\right.
$$


Consider the problem "When does there exist an integral curve in $S\left(\Lambda_{2}\right)$ ?" The answer is known if $\mu_{a, a+2} \geqq 1$. Before stating the results let us make preparations first.

Set $U_{4}={ }^{t}\left(h_{1}, \cdots, h_{a}\right), \mathfrak{a}=\left(h_{1}, \cdots, h_{a}\right) k(2,4) \subset k\left[x_{3}, x_{4}\right] . \quad$ If $I\left(\lambda_{3}\right)=R$ then $\mathfrak{a}=k(2,4)$; that is one of $h_{i}(1 \leqq i \leqq a)$ is a unit, so that (4.1.3)' can be solved easily. If $I\left(\lambda_{3}\right) \neq R$ and contains an $R$-sequence of length 4 , then $\mathfrak{a}$ contains a $k(2,4)$-sequence of length 2 , that is $k(2,4) / \mathfrak{a}$ is CohenMacaulay of dimention 0 . Hence, the $k(2,4)$-module $M=\left\{\left(v_{1}, \cdots, v_{a}\right)\right.$ $\left.\in k(2,4)^{a} \mid \sum_{i=1}^{a} v_{i} h_{i}=0\right\}$, which makes the sequence

$$
0 \longrightarrow M \longrightarrow k(2,4)^{a} \stackrel{t}{{ }^{t} U_{4}} \longrightarrow k(2,4) \longrightarrow k(2,4) / \mathfrak{a} \longrightarrow 0
$$

exact, is free of rank $a-1$ over $k(2,4)$ by Auslander-Buchsbaum's theorem. And each row vector of $V^{(0)}$ satisfying $(4.1 .3)^{\prime}$ is in $M$. Write $M_{\nu}$ for $\left\{v \in M \mid d_{\bar{e}}(v)=\nu\right\}$ where $\bar{e}=\left(\operatorname{deg} h_{1}, \cdots, \operatorname{deg} h_{a}\right)=\left(\mu_{1, a+2}, \cdots, \mu_{a, a+2}\right)$, and let $N_{p}$ be the submodule of $M$ generated by $\underset{\nu \leq p}{\oplus} M_{\nu}$. Put $\omega_{i}=\left(\mu_{i 1}\right.$, $\left.\cdots, \mu_{i a}\right)$ and $c_{i}=\operatorname{deg} h_{j}+\mu_{i j}$ (independent of $j$ ) for $0 \leqq i \leqq a$. We see $c_{0}$ $\geqq c_{1} \geqq \cdots \geqq c_{a}$.

Suppose $\left\{\begin{array}{l}c_{1}=\cdots=c_{t_{1}}=\varepsilon_{1}, \\ c_{t_{1}+1}=\cdots=c_{t_{1}+t_{2}}=\varepsilon_{2}, \quad \varepsilon_{1}>\varepsilon_{2}>\cdots>\varepsilon_{p}, \\ \quad \cdots \cdots, \\ c_{t_{1}+\cdots+t_{p-1}+1}=\cdots=c_{t_{1}+\cdots+t_{p}}=\varepsilon_{p}, \\ t_{1}+\cdots+t_{p}=a .\end{array}\right.$

Then

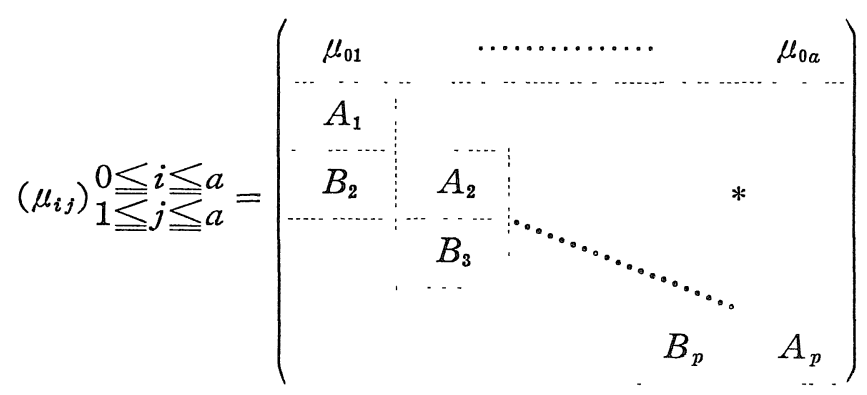

where $A_{i}$ is the $t_{i} \times t_{i}$ matrix with all entries 1 for $1 \leqq i \leqq p$ and $B_{i}$ is the $t_{i} \times t_{i-1}$ matrix with all entries 0 for $2 \leqq i \leqq p$. Let $\mathcal{M}$ denote the above matrix. We form a new $(a-1) \times a$ matrix $D=\left(d_{i j}\right) 0 \leqq i \leqq a-2$, $1 \leqq j \leqq a$ with entries in $Z$ in the following way. First put $\xi_{i}=\omega_{t_{i-1}+1}$ for 
$2 \leqq i \leqq p$, and

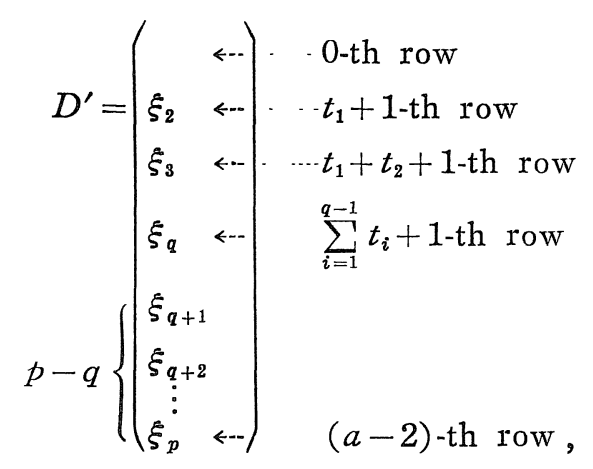

where $q$ is the largest number of $i$ such that $t_{1}+\cdots+t_{i-1}+1 \leqq(a-2)$ $-(p-i)$. Next fill each blank row of $D^{\prime}$ with the corresponding row of $\mathcal{M}$. Let $D$ be the matrix thus obtained.

Put

$$
\rho\left(\Lambda_{2}\right)=\sum_{i=0}^{a-2} d_{i+1}
$$

Lemma 4. 2. Suppose $b=1, a \geqq 2$. If $S\left(\Lambda_{2}\right)$ contains an integral curve which is not projectively Cohen-Macaulay, then

1) $\operatorname{deg} f_{0} \leqq \operatorname{deg} f_{i}$ for $1 \leqq i \leqq a+1$

2) $0 \leqq \operatorname{deg} f_{i+1}-\operatorname{deg} f_{i} \leqq 1$ for $1 \leqq i \leqq a-1$.

Proof. First note that $\operatorname{deg} f_{0} \leqq \operatorname{deg} f_{i}$ for $1 \leqq i \leqq a$ by assumption or rather by Example 2.7. Therefore, if $\operatorname{deg} f_{a+1}<\operatorname{deg} f_{0}$ we must have $\mu_{i, a+2} \leqq 0$ for $1 \leqq i \leqq a$. This implies that every nonzero $h_{i}$ must be in k. Thus $R / I(X)$ turns out to be Cohen-Macaulay, which contradicts the assumption. Hence $\operatorname{deg} f_{0} \leqq \operatorname{deg} f_{a+1}$. Since $I(X)$ is prime $f_{0}$ must be irreducible, from which 2) follows. See [6; Proposition 2.1].

Lemma 4. 3. Suppose $b=1, a \geqq 2$. There exists a scheme $X$ in $S\left(\Lambda_{2}\right)$ which does nol contain $L=\left\{\left(x_{1}: x_{2}: x_{3}: x_{4}\right) \in \mathbf{P}_{k}^{3} \mid x_{1}=x_{2}=0\right\}$ as an irreducible component if and only if rank $N_{c_{i}} \geqq a-1-i$ for all $0 \leqq i$ $\leqq a-2$. 
Proof. Let $J \subset I$ be as in Theorem 3.7. We see easily that Proj $R / J$ contains $L$ as an irreducible component. Hence Proj $R / I$ does not contain $L$ as an irreducible component if and only if $f_{a+1}\left(0,0, x_{3}, x_{4}\right)$ $\neq 0$. This is possible if and only if $\operatorname{rank} N_{c_{i}} \geqq a-1-i$ for all $0 \leqq i \leqq a-2$. With Lemmas 4.2 and 4.3 in mind we get

Proposition 4. 4 .

1) Suppose $b=1, a \geqq 3, \nu_{0} \leqq \nu_{1} \leqq \cdots \leqq \nu_{a}, \nu_{i+1}-\nu_{i} \leqq 1$ for $1 \leqq i \leqq a-1$, and $\mu_{a, a+2} \geqq 1$. Then $S\left(\Lambda_{2}\right)$ contains an integral curve if and only if

$$
\mu_{a, a+2} \leqq \rho\left(\Lambda_{2}\right) \text {. }
$$

2) Suppose $b=1, a=2$, and $\mu_{24} \geqq 1$. Then $S\left(\Lambda_{2}\right)$ contains an integral curve if and only if

$$
\mu_{14}=\mu_{24}=1 \text { and } \mu_{01}=\mu_{02} \geqq 2 \text {. }
$$

For the proof we use only Bertini's Theorem and elementary properties of determinants. Details are omitted.

Example 4.5. Suppose $r \leqq n, 2 \leqq n$, and put

$$
\Lambda_{2}=\left(\begin{array}{ccccc}
n & n & n & n+r & n+r \\
1 & 1 & 1 & r+1 & r+1 \\
1 & 1 & 1 & r+1 & r+1 \\
1 & 1 & 1 & r+1 & r+1 \\
-r+1 & -r+1 & -r+1 & 1 & 1
\end{array}\right)
$$$$
\lambda_{2}=
$$

$\left.\begin{array}{c|c|c|c|c|}-x_{2}^{n}+x_{3}^{n} & -x_{3}^{n-r} x_{4}^{r} & \left(s x_{3}+t x_{4}\right) x_{2}^{n-1}+u x_{2}^{n} & \begin{array}{c}x_{4}^{r+1} x_{2}^{n-1}-u x_{3}^{r+1} x_{2}^{n-1} \\ -x_{3}^{r+1}\left(s x_{3}+t x_{4}\right) x_{2}^{n-2}\end{array} & 0 \\ \hline x_{1} & -x_{2}+x_{3} & -x_{4} & x_{3}^{r} x_{4} & x_{4}^{r+1} \\ \hline x_{2} & x_{1} & -x_{2} & x_{3}^{r+1}-x_{4}^{r+1} & x_{3}^{r} x_{4} \\ \hline 0 & x_{2} & x_{1} & -x_{3}^{r} x_{4} & x_{3}^{r+1} \\ \hline 0 & 0 & 0 & x_{1} & x_{2}\end{array}\right)$.

Then $\rho\left(\Lambda_{2}\right)=n+1 \geqq \mu_{a, a\llcorner 2}=\mu_{35}=r+1, \lambda_{2} \cdot \lambda_{3}=0$, and 


$$
\begin{aligned}
& f_{0}=x_{1}^{3}+x_{1} x_{2}\left(2 x_{2}-x_{3}\right)-x_{2}^{2} x_{4} \\
& f_{1}=x_{1}^{2}\left(-x_{2}^{n}+x_{3}^{n}\right)+x_{1} x_{2} x_{3}^{n-r} x_{4}^{r}+x_{2}^{2} x_{3}^{n}-x_{2}^{n+2}+s x_{2}^{n+1} x_{3} \\
& \quad+t x_{2}^{n+1} x_{4}+u x_{2}^{n+2} \\
& f_{4}\left(0,0, x_{3}, x_{4}\right)=-x_{3}^{n+r} x_{4}^{2}-x_{3}^{n+r+2}-x_{3}^{n+1} x_{4}^{r+1} .
\end{aligned}
$$

One verifies directly that

$$
\text { Spec } k\left[\approx_{1}, z_{3}, \approx_{4}\right] /\left(f_{0}\left(\approx_{1}, 1, \approx_{3}, \approx_{4}\right), f_{1}\left(z_{1}, 1, \approx_{3}, z_{4}\right)\right)
$$

is irreducible reduced for a suitable choice of $s, t, u \in k$, and that Proj $R / I$ $=X$ does not have any irreducible component in $\left\{\left(x_{1}: x_{2}: x_{3}: x_{4}\right) \in \mathbf{P}_{k}^{3}\right.$ $\left.! x_{2}=0\right\}$. Thus $x$ is an integral curve for a suitable choice of $s, t, u \in k$.

Remark 4.6. The curves obtained in Proposition 4.4 have singularities in many cases. In fact we can prove the following:

(\#) Let $g_{0}, \cdots, g_{a-2}$ be a free basis for $M$, and suppose $d_{\bar{e}}\left(g_{0}\right)$ $\geqq d_{\bar{e}}\left(g_{1}\right) \geqq \cdots \geqq d_{\bar{e}}\left(g_{a-2}\right)$. If $d_{\bar{e}}\left(g_{0}\right)<c_{0}$ and $g_{0} \notin N_{c_{1}}$, then the integral curves in $S\left(\Lambda_{2}\right)$ must have singularities.

\section{References}

[1] Buchsbaum, D. and Eisenbud, D., What makes a complex exact? Journal of Algebra, 25 (1973), 259-268.

[2] Briançon, J. et Galligo, A., Déformations de points $\boldsymbol{R}^{2}$ ou $\mathbb{C}^{2}$, Astérisque, 7 et 8 (1973), 129-138.

[ 3 ] Galligo, A., A propos du théorèm de préparation, Springer Lecture Notes, 409(1973), 543-579.

[4] - Théorèm de division et stabilité en géometrie analytique locale, Ann. Inst. Fourier, Grenoble 29 (1979), 107-184.

[5] Grauert, H., Über die Deformation isolierter Singularitäten analytischer Mengen, Inventiones Math., 15 (1972), 171-198.

[6] Gruson, L. et Peskine, C., Genre des courbes de l'espace projectif, Proceedings, Troms $\phi$, Norway 1977, Springer Lecture Notes 687.

[7] Hironaka, H., Bimeromorphic smoothing of complex analytic spaces, Preprint, Warwick University, 1971.

[8] Lectured by Hironaka, H., notes by Urabe, T., "Introduction to analytic spaces" (In Japanese) Asakura Publ. Comp. 1982.

[ 9] Urabe, T., On Hirona's monoideal. Publ. RIMS, Kyoto Univ., 15 (1979), 279-287. 